\title{
Transnasal endoscopic excision of a dermoid cyst of the nasal septum in an infant: A case report
}

\section{Sónia P. Martins ${ }^{1,2 *}$, Pedro Canão ${ }^{3}$, João Pinto ${ }^{1,2}$, Ricardo P. Vaz ${ }^{1,2,5}$, Carla P. Moura ${ }^{1,6,7}$, Jorge Spratley ${ }^{1,2}$}

${ }^{1}$ Department of Otorhinolaryngology, University Hospital Centre of São João, Alameda Professor Hernâni Monteiro, 4200- 319, Oporto, Portugal. ${ }^{2}$ Unit of Otorhinolaryngology, Department of Surgery and Physiology, University of Oporto Faculty of Medicine, Alameda Professor Hernâni Monteiro, 4200-319, Oporto, Portugal.

${ }^{3}$ Department of Pathology, University Hospital Centre of São João, Alameda Professor Hernâni Monteiro, 4200-319, Oporto, Portugal.

${ }^{4}$ Unit of Anatomy - Department of Biomedicine, Faculty of Medicine, University of Porto, Alameda Professor Hernâni Monteiro, 4200-319, Porto, Portugal.

${ }^{5}$ Center for Health Technology and Services Research (CINTESIS), Rua Dr. Plácido da Costa, 4200-450, Oporto, Portugal.

${ }^{6}$ Department of Genetics, Centro Hospitalar Universitário São João, Faculty of Medicine of University of Oporto, Alameda Professor Hernâni Monteiro, 4200-319, Oporto, Portugal.

${ }^{7}$ I3S, Instituto de Investigação e Inovação em Saúude, Institute Research and Innovation Health, University of Oporto, Portugal.

* Corresponding Author: Sónia P. Martins, Department of Otorhinolaryngology, University Hospital Centre of São João, Alameda Professor Hernâni Monteiro, 4200- 319, Oporto, Portugal, Unit of Otorhinolaryngology, Department of Surgery and Physiology, University of Oporto Faculty of Medicine, Alameda Professor Hernâni Monteiro, 4200-319, Oporto, Portugal.

Received date: 31 January 2022; Accepted date: 10 February 2022; Published date: 17 February 2022

Citation: Martins SP, Canão P, Pinto J, Vaz RP, Moura CP, et al. (2022) Transnasal endoscopic excision of a dermoid cyst of the nasal septum in an infant: A case report. J Med Case Rep Case Series 3(02): https://doi.org/10.38207/JMCRCS/2022/JAN03020514

Copyright: C 2022 Sónia P. Martins. This is an open-access article distributed under the terms of the Creative Commons Attribution License, which permits unrestricted use, distribution, and reproduction in any medium, provided the original author and source are credited.

\footnotetext{
Abstract

Dermoid cysts are congenital, ectoderm and mesoderm derived lesions of the median line. Their location is variable, and the treatment consists of the surgical removal of the lesion.

We report a case of a nasal dermoid cyst presenting with an uncommon location and discuss surgical outcomes.

The occurrence of dermoid cysts in unusual locations, such as the nasal septum, must be considered in the differential diagnosis of nasa obstruction in the young child. The surgical approach of these deeply located lesions is a challenge, particularly in small children, given the small size of nasal anatomical structures.
}

Keywords: Nasal obstruction; Congenital abnormality; Dermoid cyst; Surgical procedure, Nasal septum

\section{Introduction}

Congenital midline nasal masses have a reported incidence of 1 in 20000-40000 patients, in which nasal dermoid cysts (NDC) are among the most common [1]. Embryologically, nasal dermoids originate from an anomaly of the anterior neuropore that is believed to result from failed involution of the neuroectoderm within the prenasal space. These lesions are lined with stratified squamous epithelium and may contain adnexal structures such as hair or sebaceous glands [2,3].

NDC is more common in the nasofrontal area and may be associated with a pit [4]. If not recognized, NDC can cause recurrent infection, midfacial deformities, and nasal obstruction [5].

\section{Case report}

A 9-month-old infant was evaluated in the outpatient clinic due to bilateral nasal obstruction since birth. There was no history of complications during pregnancy and birth, and the child developed normally in the neonatal period. Parents denied dyspnea, feeding
In this report, we describe a case of a dermoid cyst presenting in an exceedingly rare location - the nasal septum. Rapid recognition of the pathology and appropriate treatment are essential, both in promoting normal development and preventing potential complications.

The objectives of this work were to integrate the current case in the literature regarding NDC of the nasal septum and to discuss new approaches and surgical options in NDC of the nasal septum diagnosed in the pediatric age.

difficulties, and infectious complications. On physical examination, there were no facial deformities or lesions. Nasal endoscopy showed a bilateral bulging of the mucosa in the posterior septal region that occupied a significant portion of both nasal cavities (figure 1). 
Computed tomography (CT) and magnetic resonance imaging (MRI) demonstrated a well-circumscribed oval lesion measuring 10x8 mm in the posterior nasal septum without extension into the intracranial cavity (figure 2). This lesion consisted of a solid mass with a little cystic component suggestive of lipomatous nature.

Consent for an oral and/or transpalatal approach was obtained preoperatively. Full removal of the mass was accomplished exclusively by transnasal endoscopic surgery followed by stenting of both nasal cavities using a previously cut orotracheal tube (figure 3 ). The stents were removed 14 days after surgery.

The histopathological examination confirmed the diagnosis of the dermoid cyst, characterized by a lining of stratified squamous epithelium associated with pilosebaceous and eccrine adnexal structures (figure 4).

No complications occurred post-operatively. Currently, two years after surgery, the nasal mucosa is completely healed, without signs of recurrence and the airway patency is excellent (figure 5).

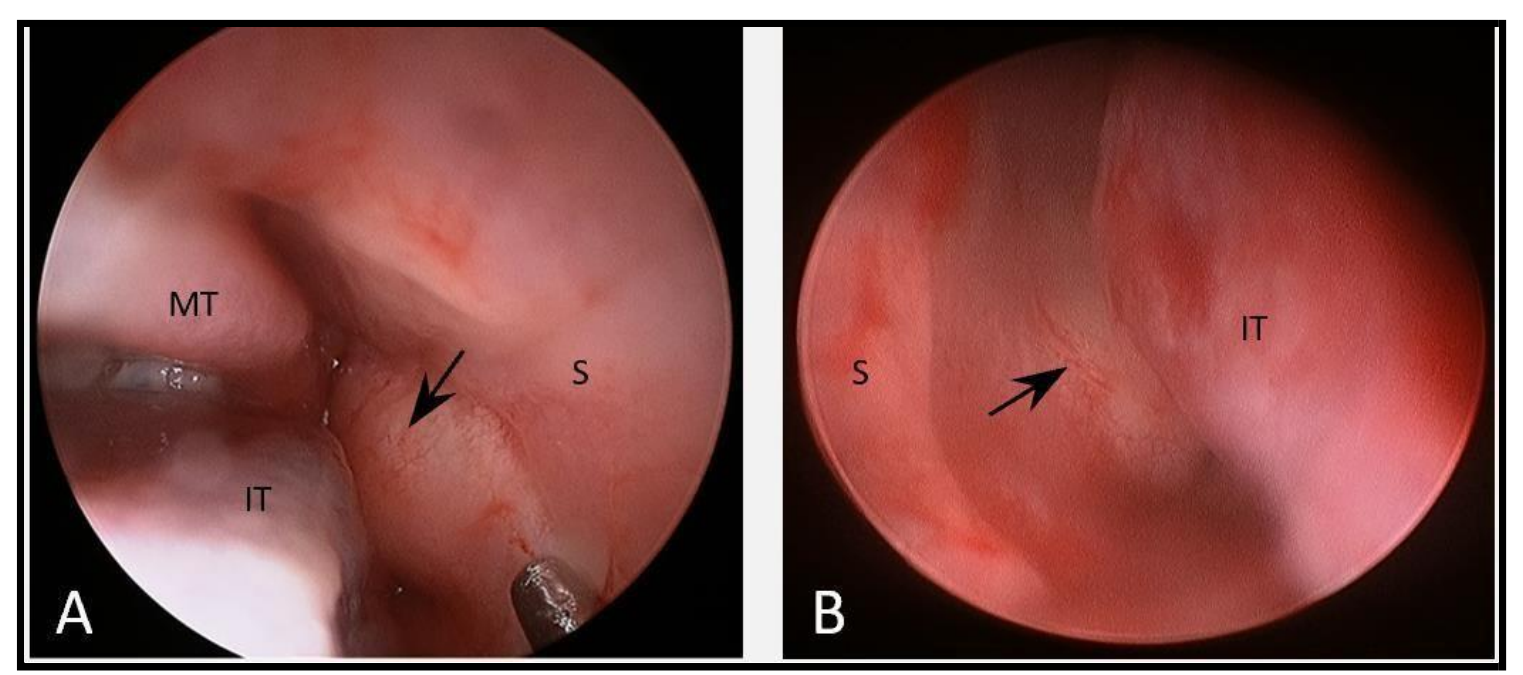

Figure 1: Nasal endoscopy showing a round mass in the posterior septal region that obstructs both nasal cavities (arrow). A) right nasal cavity; B) left nasal cavity. S - septum, IT - inferior turbinate, MT - middle turbinate.

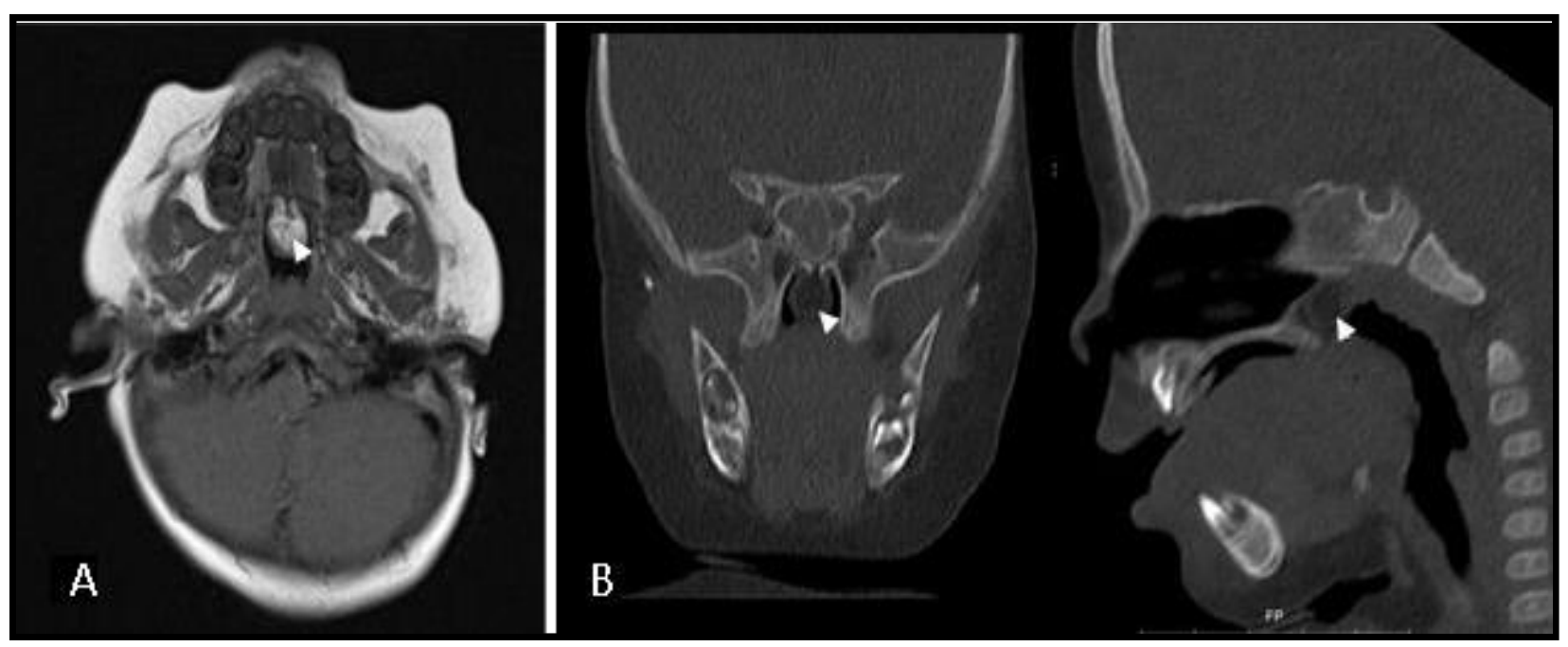

Figure 2: Dermoid cyst imaging showing a round and well-circumscribed mass located in the posterior septal region (arrowhead). A) Magnetic resonance imaging, B) Computed tomography.

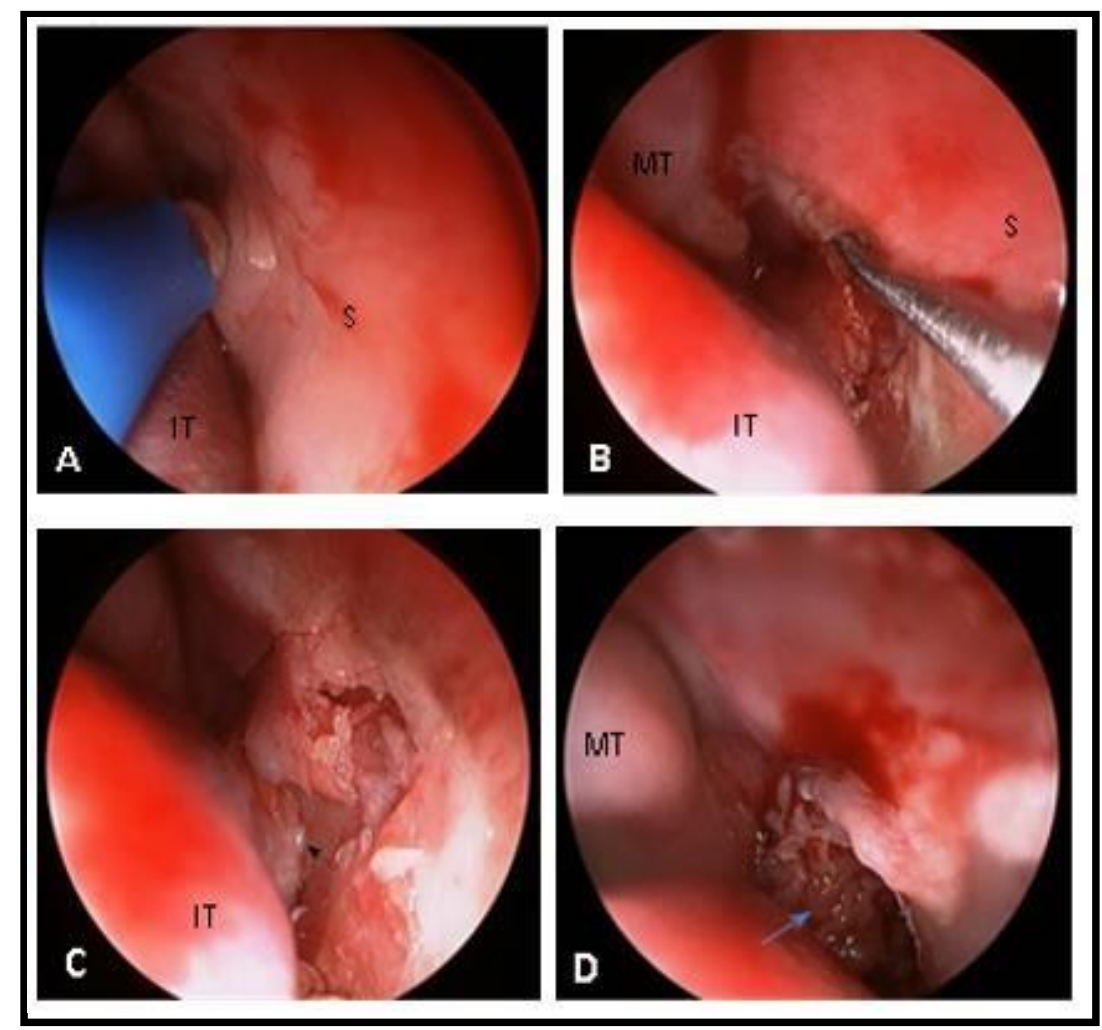

Figure 3: Photographs taken during the transnasal endoscopic surgery (right nasal cavity). Each step was performed on both sides. A) Mucosal incision anterior to the lesion; B) Dissection in the subperiosteal plane along the septum, in a posterior direction; C) Identification of the posterior border of the vomer (arrowhead); D) Lesion after total individualization (arrow); S - septum, IT - inferior turbinate, MT - middle turbinate. 

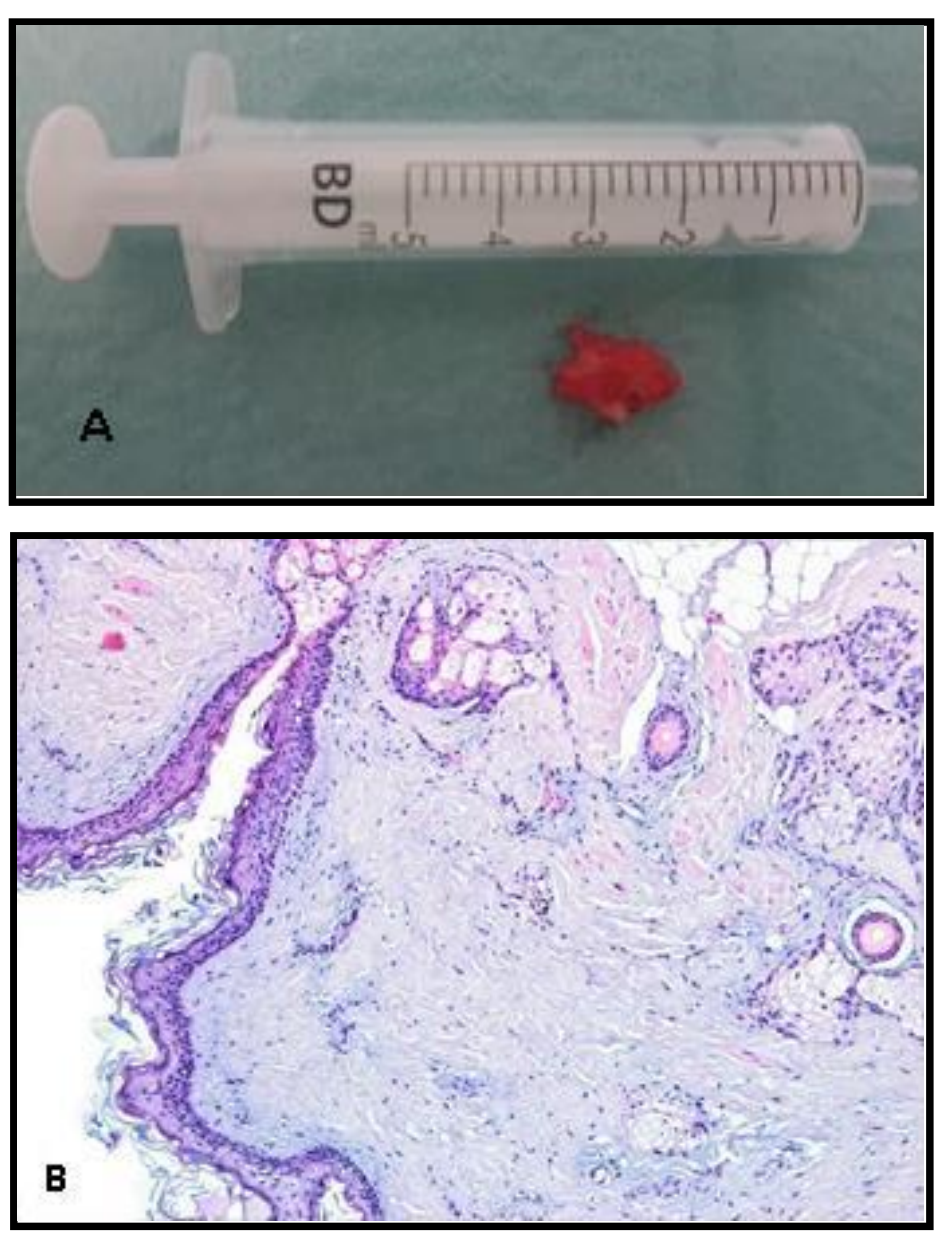

Figure 4: Dermoid Cyst. A) Macroscopic aspect; B) Histology: cystic lesion composed of fibrous wall lined by keratinizing squamous epithelium with pilosebaceous units and scattered eccrine sweat glands (HE, x100).

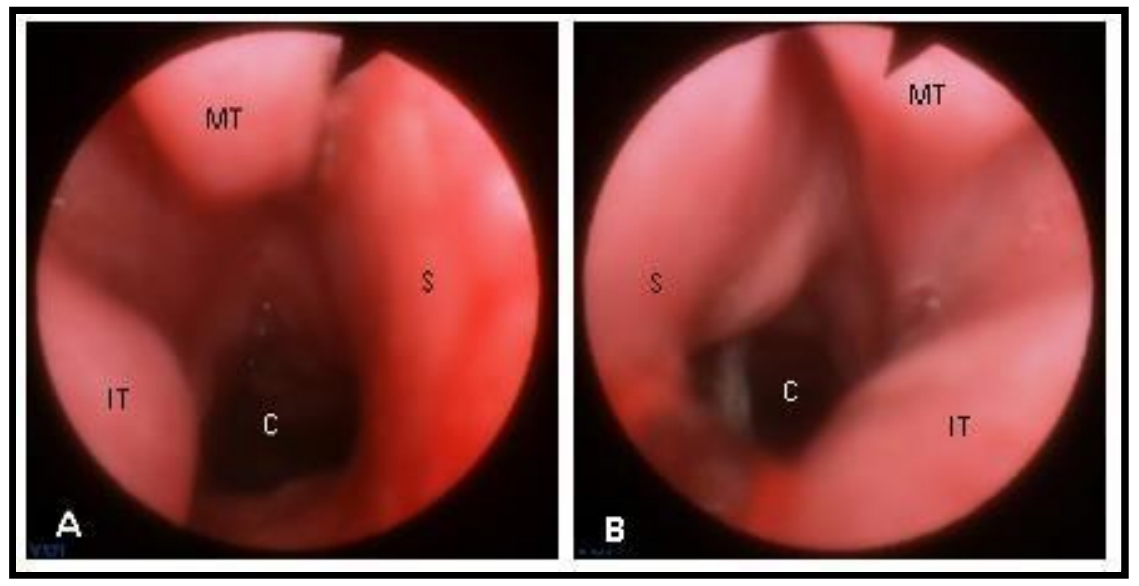

Figure 5: Nasal endoscopy six months after surgery. Both nasal cavities are patent. A) right nasal cavity; B) left nasal cavity. S - septum, IT inferior turbinate, MT- middle turbinate, $\mathrm{C}$ - choanae

\section{Discussion}

Dermoid cysts located in the nasal septum are exceedingly rare. In fact, few cases were reported in the literature. The oldest case dates back to 1941 when Juers and Louisville reported a dermoid cyst of the nasal septum that was excised intranasally in a patient of 19 years of age [6]. One of the first case series of NDC was published in the late seventies, in which only 5 of 21 nasal dermoid cases were located in the nasal septum [7]. Additionally, other authors described a discharging pit as the first manifestation of NDC in the nasal septum diagnosed in adolescence and early adulthood $[8,9]$. However, in our case report, NDC has diagnosed in a 9-month-year-old child in which nasal obstruction was the main symptom. No pit was recognized when exploring the nose, the nasal and oral cavities, and the pharynx of the child. Prompt investigation and treatment was carried out to promote a good craniofacial development
Imaging is important to assist in the diagnosis, to evaluate the extension of the lesion, and to exclude intracranial involvement which is present in up to $45 \%$ of nasal dermoid cases [2,10]. MRI is the modality of choice given its lack of ionizing radiation and excellent characterization of soft tissues using different sequences. CT has the well-known disadvantages of ionizing radiation, although it gives complimentary information with a better definition of bone anatomy which is an asset to surgical planning $[2,11]$.

Complete surgical excision of the lesion is mandatory to prevent recurrence and to prevent infection. The surgical strategy for excision of the nasal dermoid should provide access to the cyst and allow adequate access to the skull base if required [2]. The choice of the surgical approach depends on the location of the cyst and the presence or absence of intracranial extension [3]. 
Several approaches have been described, such as endoscopic transnasal resection, open rhinoplasty, and transcranial approach $[2,11]$. The transcranial approach, combined or not with transnasal endoscopic surgery, is reserved for lesions with significant or indeterminate intracranial extension [11]. In recent years, this approach has been replaced by the transnasal endoscopic approach $[\mathbf{2 , 1 1 - 1 4 ]}$. The external rhinoplasty approach is used when the cyst lies within the lower soft tissue vault of the nose [11]. The transnasal endoscopic excision of NDC is performed when the cyst is located within the nasal cavity with minimal cutaneous involvement [14], which happened to be the current case.

When planning for NDC surgical excision, the four criteria proposed by Pollock should be met [15]. First, the surgical approach should permit access to all midline cysts and should readily permit medial and lateral osteotomies if required. Second, the surgical exposure should favor the rapid repair of cribriform defects and control of cerebrospinal fluid leak if it develops. Third, the ideal method would facilitate the reconstruction of the nasal dorsum. Fourth, the chosen surgical procedure should result in an acceptable postoperative scar. The authors predicted that the transnasal endoscopic approach would provide the best access to the cyst, which met the first Pollock criterion. Regarding the second criterion, the preoperative imaging did not show intracranial extension. However, endoscopic sinus surgery is associated with cranial basefistulaecomplications. So,thesurgeon's experience in dealing with potential intra-operative complications of endoscopic sinus surgery was a factor that favored this approach. In

\section{Conclusions}

A nasal dermoid cyst of the nasal septum is a rare entity that can lead to life-threatening nasal obstruction. The clinical manifestations may resemble choanal atresia. Differential diagnosis in the newborn includes other midline nasal masses/cysts, such as encephalocele and nasal glioma. MRI is the best image modality to exclude intracranial involvement. Complete surgical excision is paramount to prevent

\section{References}

1. Hughes GB, Sharpino G, Hunt W, Tucker HM (1980) Management of the congenital midline nasal mass: a review. Head Neck Surg. 2(3): 222-33.

2. Moses MA, Green BC, Cugno S, Hayward RD, Jeelani NU, et al. (2015) The management of midline frontonasal dermoids: a review of 55 cases at a tertiary referral center and a protocol for treatment. Plast Reconstr Surg. 135(1): 187-196.

3. Rodriguez DP, Orscheln ES, Koch BL (2017) Masses of the Nose, NasalCavity, andNasopharynx in Children. Radiographics. 37(6): 1704-30.

4. Sessions RB (1982) Nasal dermal sinuses-new concepts and explanations. The Laryngoscope. 92(8 Pt 2 Suppl 29): 1-28.

5. Frodel JL, Larrabee WF, Raisis J (1989) The nasal dermoid. the current case, the third and fourth criteria did not apply since the cyst was in the posterior part of the nasal septum without the involvement of the nasal pyramid structures.

Also, our case met the requirements described by Weiss et al. for a successful endoscopic surgical approach: 1) open cavity, 2) relatively avascular planes, and 3) predictable anatomic landmarks [14]. The transnasal endoscopic approach has other important advantages: it is minimally invasive; it is also suitable for intracranial NDC and allows a subperiosteal dissection of the cyst walls from the bony structures [2]. In fact, the non-invasive feature of the NDC makes the plane of dissection easy to manipulate, while preserving the underlying neural and vascular structures [12]. The surgeon's main concern was that the small size of the infant nasal cavities could make it difficult to safely handle the instruments inside the nose. Hence, during preoperative planning, the authors considered an alternative surgical approach - a transoral or transpalatal approach. As far as the authors know, no literature exists about a transoral or transpalatal excision of dermoid cysts.

However, these approaches were thought preoperatively in case of failure of the transnasal endoscopic technique. Given the posterior location of the cyst in the nasal septum, the cyst would be removed transorally, with or without a section of the soft palate, to ensure complete removal of the lesion. In the present case, the transnasal endoscopic approach had resulted and the child is currently asymptomatic with no signs of recurrence.

recurrence and infection. Diagnosis is confirmed by histologic examination.

5. Funding: This research did not receive any specific grant from funding agencies in the public, commercial, or non-profit sector.

6. Declaration of competing interest: The authors report no conflicts of interest related to this study

Otolaryngol Head Neck Surg. 101(3): 392-396.

6. Juers AL, Louisville KY (1941) Dermoid cyst of the nasal septum: Intranasal Excision. Arch Otolaryngol. 33(5): 851-852.

7. McCaffrey TV, McDonald TJ, Gorenstein A (1979) Dermoid cysts of the nose: review of 21 cases. Otolaryngol Head Neck Surg. 87(1): 52-59.

8. Choi YD, Park ES, Kang MS (2012) A nasal dermal sinus cyst involving the nasal septum. J Craniofac Surg. 23(6): e636-8.

9. Lee DH, Yoon TM, Lee JK, Lim SC (2018) Dermoid cyst of nasal septum in an adult patient: A case report. Medicine (Baltimore). 97(45): e13028.

10. Bradley PJ (1982) Results of surgery for nasal dermoids in children. J Laryngol Otol. 96(7): 627-33. 
11. Herrington H, Adil E, Moritz E, Robson C, Perez-Atayde A, et al. (2016) Update on current evaluation and management of pediatric nasal dermoid. The Laryngoscope. 126(9): 2151-60.

12. Düz B, Secer HI, Tosun F, Gonul E (2007) Endoscopic endonasal resection of a midline intradural frontobasal dermoid tumour. Minim Invasive Neurosurg. 50(6): 363-6.

13. Re M, Tarchini P, Macrì G, Pasquini E (2012) Endonasal endoscopic approach for intracranial nasal dermoid sinus cysts in children. Int J Pediatr Otorhinolaryngol. 76(8): 1217-22.

14. Weiss DD, Robson CD, Mulliken JB (1998) Transnasal endoscopic excision of midline nasal dermoid from the anterior cranial base. Plast Reconstr Surg. 102(6): 2119-23.

15. Pollock RA (1983) Surgical approaches to the nasal dermoid cyst. Ann Plast Surg. 10(6): 498-501. 